\title{
Association between Tooth Loss and the Development of Mild Memory Impairment in the Elderly: The Fujiwara-kyo Study
}

\author{
Nozomi Okamoto $^{\mathrm{a}, *}$, Masayuki Morikawa ${ }^{\mathrm{b}, \mathrm{c}}$, Kimiko Tomioka ${ }^{\mathrm{a}}$, Motokazu Yanagi ${ }^{\mathrm{d}}$, Nobuko Amano ${ }^{\mathrm{d}}$ \\ and Norio Kurumatani ${ }^{\mathrm{a}}$ \\ ${ }^{a}$ Department of Community Health and Epidemiology, Nara Medical University, Japan \\ ${ }^{\mathrm{b}}$ Mie Prefectural Mental Care Center, Japan \\ ${ }^{\mathrm{c}}$ Department of Psychiatry, Nara Medical University, Japan \\ ${ }^{\mathrm{d}}$ Department of Food and Nutrition, Tezukayama University, Japan
}

Accepted 23 September 2014

\begin{abstract}
.
Background: Tooth loss may be a modifiable risk factor for memory disorders, but the causal relationship has not been evaluated sufficiently.

Objective: This 5-year prospective cohort study investigated the effect of tooth loss on the development of mild memory impairment (MMI) among the elderly.

Methods: Data are from the baseline and follow-up examinations of 2,335 community residents who were cognitively intact at baseline. The number of remaining teeth at baseline was classified as zero, 1-8, 9-16, 17-24, and 25-32. The main outcome for the analysis was the development of MMI at follow-up.

Results: After adjustment for potential confounding factors in multivariable logistic regression analysis, the odds ratio of per 1 tooth loss at baseline was 1.02 (95\% confidence interval, 1.00-1.03). The odds ratio of edentulism for MMI was 2.39 (1.48-3.86) compared to $25-32$ teeth. The odds ratio of becoming edentulous compared to retaining $1-8$ teeth in the $1-8$ teeth group at baseline was 4.68 (1.50-14.58).
\end{abstract}

Conclusion: Tooth loss predicts the development of MMI among the elderly.

Keywords: Alzheimer's disease, dementia, memory, periodontal disease, prospective cohort study, tooth loss

\section{INTRODUCTION}

Epidemiological studies have reported that tooth loss is associated with Alzheimer's disease (AD) and dementia. Analysis of twins showed that tooth loss before 35 years of age was a significant risk factor for AD [1]. The Nun study, a longitudinal study of Roman Catholic nuns, found that fewer teeth (9 or less) were associated with an increased risk of dementia [2].

\footnotetext{
*Correspondence to: Nozomi Okamoto, Department of Community Health and Epidemiology, Nara Medical University, Shijo-cho 840, Kashihara City, Nara 6348521, Japan. Tel.: +81 74429 8841; Fax: +81 74429 0673; E-mail: onozomi@ naramed-u.ac.jp.
}

A 5-year prospective cohort study among patients with type 2 diabetes showed that having no teeth was significantly associated with the risk of dementia and cognitive decline [3]. A significant relationship between natural teeth and preserved cognitive functioning [4], significant relationships between fewer teeth (10 or less) and lower Wechsler Adult Intelligence Scale subtest scores [5], and a significant correlation between fewer teeth and worse episodic memory [6] have been reported in cross-sectional studies. Tooth loss is considered the ultimate outcome of periodontal disease [7]; thus, it has been hypothesized that the biological bases for the association between tooth loss, $\mathrm{AD}$, and dementia may include periodontal 
disease-derived inflammatory molecules, bacteria, and bacterial products that enhance neuroinflammation [8]. Tooth loss and periodontal disease may be putative modifiable risk factors for $\mathrm{AD}$ and dementia, and the identification of modifiable risk factors represents an important preventive strategy for $\mathrm{AD}$ and dementia.

A community-based survey reported that, among persons with mild memory impairment (MMI) [9], defined using the Mini-Mental State Examination (MMSE), 21.2\% progressed to illnesses with dementia, including $\mathrm{AD}(10.6 \%)$, vascular dementia (4.8\%), or other types of dementia $(5.8 \%)$, over a period of 5 years. These individuals represent a high-risk population for $\mathrm{AD}$ and dementia, although they have normal general cognitive function and no impairment of the activities of daily living (ADL). MMI is comparable to mild cognitive impairment (MCI) [10], which is an isolated memory disorder that can precede dementia [11]. In previous cross-sectional studies, we found that a low number of teeth was related to an increased prevalence of MMI [12, 13]. We hypothesized that tooth loss may be a risk factor for a preclinical stage of $\mathrm{AD}$ and dementia. To date, only a small number of cohort studies $[2,3,14,15]$ have investigated the causal relationships between tooth loss and memory or cognitive decline. When examining the effect of tooth loss on memory or cognitive decline, it is desirable to exclude the possible effect of the onset of impaired cognitive function during a cohort study on the onset of tooth loss. In order to achieve this, the current study chose MMI instead of cognitive impairment as our outcome variable. Impaired cognitive function induces poor oral health and tooth loss. MMI, however, is less likely to induce poor oral health relative to cognitive dysfunction due to its independence from impaired ADL. The purpose of this prospective cohort study was to examine the effect of having a low number of teeth on the development of MMI.

\section{MATERIALS AND METHODS}

\section{Selection of subjects}

This study was approved by the Medical Ethics Committee of Nara Medical University.

We used data from the baseline (in 2007 and the beginning of 2008) and follow-up (in 2012) examinations of the Fujiwara-kyo study. Written informed consent was obtained from each of the subjects prior to their participation in the baseline and follow-up examinations. The Fujiwara-kyo study $[16,17]$ is an ongoing cohort study of volunteer men and women who, at baseline, were independent elderly residents of Nara Prefecture aged 65 years or over and able to walk unassisted.

Figure 1 shows the selection of the subjects. Among the 4,206 persons who participated in the baseline examination, 3,696 cognitively intact individuals were enrolled in the current study. By the time of the 2012 follow-up examination, 159 subjects had died, 42 had moved away, 15 had been admitted to an institution, 260 were hospitalized or were receiving medical treatment, and 734 were non-responders. Thus, 2,486 participants underwent the follow-up examination. Among the 2,486 participants, we excluded 111 individuals who had cognitive impairment at follow-up and 40 individuals who were suspected of pseudo-MMI induced by depression at follow-up. Ultimately, the 2,335 subjects who were identified as being cognitively intact or MMI at follow-up were included in the current study.

\section{Fujiwara-kyo study initiated}

Of the 4206 participants in the baseline examination, 3696 individuals who were cognitively intact were enrolled in the current study.

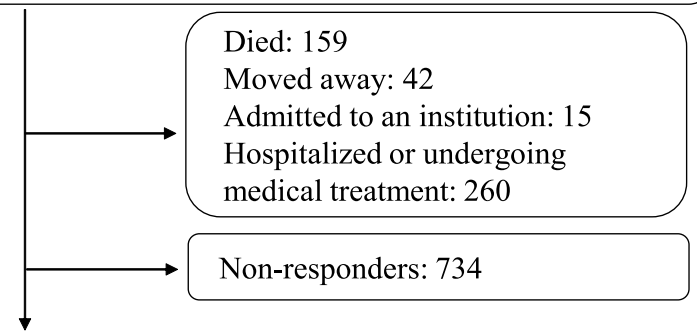

2012 Follow-up examination

2486 underwent the follow-up examination $(67.3 \%$, $2486 / 3696)$

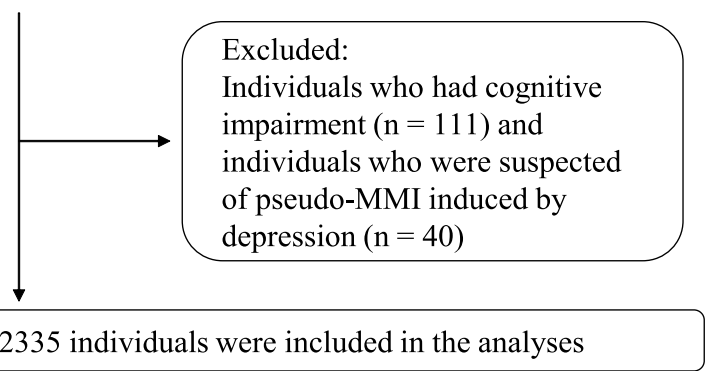

Abbreviation: MMI, mild memory impairment.

Fig. 1. Selection of subjects. 
Study design

This was a 5-year prospective cohort study.

\section{Definition of cognitive mental status}

MMI was defined according to Ishikawa et al. as follows [9]: 1) no impairment of the ADL; 2) normal general cognitive function, as determined using the MMSE (score range: 0-30) $\geq 24$ [18]; 3) objective memory impairment, assessed by the three-word delayed recall test in the MMSE (Recall, score range: $0-3) \leq 1$; and 4) absence of dementia or depression, diagnosed by geriatric neuropsychiatrists according to the Diagnostic and Statistical Manual of Mental Disorders, 3rd edition, revised criteria [19]. Higher MMSE and Recall scores indicate better cognitive function.

In our study, the Tokyo Metropolitan Institute of Gerontology Index of Competence [20], a selfadministered questionnaire, was used to evaluate the ADL. The basic and instrumental ADL of all subjects were independent at baseline. The MMSE was carried out at the baseline and follow-up surveys by a psychiatrist, clinical psychologists, or graduate students who majored in psychology and were trained by a psychiatrist. The impairment of remote memory was evaluated using the Recall test. The presence or absence of depression was evaluated using the Geriatric Depression Scale short version (GDS) (score range, 0-15; cut-off score, 5/6) [21]. The GDS was included in a self-administered questionnaire survey. Higher total scores indicate a greater number of depressive symptoms. The subjects were defined as follows: "cognitively intact," an MMSE score of 24 or more plus a Recall score of 2 or 3; "MMI status," an MMSE score of 24 or more, plus a Recall score of 0 or 1 , plus a GDS score of 5 or lower (depression-free); "suspected of pseudo-MMI induced by depression," an MMSE score of 24 or more, plus a Recall score of 0 or 1 , plus a GDS score of 6 or more; and "cognitively impaired," an MMSE score of 23 or lower.

We identified the absence of dementia or depression operationally using the MMSE and GDS screening tests because of the need to shorten the time required for the assessments in such a large-scale communitybased survey. The prevalence of MMI in our study at baseline (2.9\%: 121/4206) [13] was similar to the prevalence of Japanese community-based MCI [22, 23] identified using the criteria of Petersen et al. [11]. Therefore, the MMI subjects in this study are considered to be approximate to individuals with MCI.

\section{Dental examinations}

Dental examinations were carried out at the baseline and follow-up surveys by two dentists calibrated as to the techniques using the blinded single observer method, with both the dentist and the subject in a sitting position under artificial lighting.

The remaining teeth were defined as healthy, carious, or treated (including crowned, inlay, and abutment teeth for bridge work), inclusive of completely erupted third molars. Root tips and very loose teeth that were indicated for extraction were not included as remaining teeth. We divided the subjects into 5 groups based on the number of remaining teeth at baseline: an edentulous group and groups with 1-8, 9-16, 17-24, and 25-32 teeth. Changes in the number of teeth were defined as baseline number minus follow-up number. A decrease of a tooth category was defined as a 1 or more decrease of tooth category at follow-up (e.g., changing from the 17-24 teeth group at baseline to the 9-16 teeth group at follow-up).

The Community Periodontal Index (CPI) code of the World Health Organization (WHO) [24] was recorded to evaluate the depth of the periodontal pockets. The oral cavity was divided into 6 prescribed segments, and each segment was subjected to further examination if it contained 2 or more remaining teeth that did not require extraction. The prescribed 10 representative teeth (tooth position: 11, 16, 17, 26, 27, 31, 36, 37, 46 , and 47) in the 6 segments were examined at 4 sites on every tooth using a WHO probe. Each segment was assigned to 1 of 5 code levels (code 0 , healthy; code 1 , gingival bleeding after probing; code 2 , calculus present in the periodontal pocket; code 3 , periodontal pocket $4-5 \mathrm{~mm}$ deep; and code 4 , periodontal pocket at least $6 \mathrm{~mm}$ deep), or an ineligible segment (segment having 1 or zero remaining teeth). The highest code level among the 6 segments examined was regarded as the maximum CPI code for the individual.

The weighted kappa coefficients of interobserver agreement regarding the tooth category and CPI code were 0.86 and 0.55 , respectively $(n=30)$.

\section{Measurements of potential confounding factors for tooth loss, $A D$, and dementia}

\section{Assessment of education length and lifestyle}

The subjects were asked in a self-administered questionnaire about their education level (less than 12 years; 12 or more years), drinking frequency (hardly drink; drink on at least 1 day a week), and smoking habits (never and ex-smoker; current smoker). 
Measurement of blood pressure, interview for disease history, and drawing blood

After sitting quietly for more than $5 \mathrm{~min}$, blood pressure was determined twice at an interval of $30 \mathrm{~s}$ using an automatic blood-pressure manometer (ES-P2100; TERUMO Co., Tokyo, Japan). An average of two measurements was used in the analyses. Each subject underwent an interview to record any history of disease (cancer, myocardial infarction, cerebrovascular disease, diabetes mellitus, hypertension, or dyslipidemia) and current medication. Blood samples were collected from an antecubital vein after an overnight fast.

\section{Definitions of diabetes mellitus, hypertension, and dyslipidemia}

Cancer, myocardial infarction, and cerebrovascular disease were determined by medical history and current medication. Diabetes mellitus was defined by medical history, current antidiabetic medication, and/or by one of the following biochemical test results according to the guidelines of the Japan Diabetes Society [25]: fasting plasma glucose level $\geq 126 \mathrm{mg} / \mathrm{dL}$ or hemoglobin A1c level $\geq 6.5 \%$. Hypertension was defined according to the Japanese Society of Hypertension criteria [26] as follows: medical history, current use of antihypertensive medicine, and/or systolic/diastolic blood pressures $140 / 90 \mathrm{mmHg}$ or greater. Dyslipidemia was defined by medical history, current lipid-lowering medications, and/or by one of the following biochemical test results according to the Japan Atherosclerosis Society guidelines [27]: triglycerides $\geq 150 \mathrm{mg} / \mathrm{dL}$ or low-density lipoprotein cholesterol $\geq 140 \mathrm{mg} / \mathrm{dL}$ or high-density lipoprotein cholesterol $<40 \mathrm{mg} / \mathrm{dL}$.

\section{Statistical analysis}

Statistical analysis was performed using SPSS (version 17.0; SPSS Japan, Inc., Tokyo, Japan). Two-tailed $p$ values were calculated in all of the analyses. The alpha level of significance was set at 0.05. Baseline characteristics were compared between subjects available for analysis and subjects excluded from the analysis using the chi-square or Mann-Whitney test. Differences in the characteristics of the 5 tooth groups at baseline were analyzed using the chi-square or Kruskal-Wallis test. Trend tests were performed using the Mantel-extension method (for categorical variables) or Jonckheere test (for continuous variables).
The age- (65-69 years, 70-74 years, 75-79 years, 80-84 years, and 85 years or more) and genderadjusted cumulative incidence of MMI was calculated by means of the direct method (reference: $25-32$ teeth group) and compared by using logistic regression analysis.

Multivariate-adjusted odds ratios (ORs) and 95\% confidence intervals (CIs) were determined using logistic regression analyses (forced entry method). The development of MMI was used as a dependent variable. The number of remaining teeth at baseline (continuous variable), tooth categories at baseline, and $\mathrm{CPI}$ codes at baseline were used as independent variables. Dental variables were added separately to each model. The effects of age (continuous variable), gender, MMSE-total, Recall, GDS, and education length were controlled in Model 1. The effects of the confounding factors used in Model 1 and the other confounding factors such as smoking, drinking, disease history, systolic and diastolic blood pressure at follow-up, and cerebrovascular disease for which treatment was initiated during the follow-up period were controlled in Model 2. Goodness of fit was assessed on the basis of the technique of Hosmer and Lemeshow.

Changes in the number of teeth and decreases of tooth category at follow-up were also used as independent variables in other logistic analyses. Adjusted ORs for a 1 or more category decrease were calculated separately for each of the tooth categories at baseline.

\section{RESULTS}

Comparison of baseline characteristics between the 2,335 subjects available for the analysis and the 1,361 subjects excluded from the analysis

At baseline, the median age (71.0 years for available versus 73.0 years for excluded), frequency of edentulism (7.6\% versus $10.6 \%$ ), Recall score of 2 points (24.3\% versus $32.3 \%)$, and education length less than 12 years $(21.8 \%$ versus $34.8 \%)$ were significantly greater in the excluded subjects. The median number of teeth (23.0 versus 20.0) was significant less in the excluded subjects.

\section{Relationship between tooth loss and MMI}

Table 1 shows the characteristics of the 2,335 subjects by the number of remaining teeth at the baseline survey. Significant associations were found between having fewer teeth and older age, lower MMSE total score, greater frequency of CPI code 4 (worse 
Table 1

Characteristics of 2,335 subjects by the number of remaining teeth at the baseline survey

\begin{tabular}{|c|c|c|c|c|c|c|c|}
\hline \multirow[t]{2}{*}{ Variables } & \multicolumn{7}{|c|}{ Number of remaining teeth } \\
\hline & $\begin{array}{c}25-32 \\
n=893\end{array}$ & $\begin{array}{c}17-24 \\
n=707\end{array}$ & $\begin{array}{c}9-16 \\
n=314\end{array}$ & $\begin{array}{c}1-8 \\
n=244\end{array}$ & $\begin{array}{l}\text { edentulous } \\
\quad n=177\end{array}$ & $P$ value* & $P$ value $^{\dagger}$ \\
\hline \multicolumn{8}{|l|}{ Baseline characteristics } \\
\hline Number of remaining teeth & $27.0(2.0)$ & $22.0(4.0)$ & $12.0(4.0)$ & $5.0(5.0)$ & $0(0)$ & $<0.001$ & $<0.001$ \\
\hline \multicolumn{8}{|l|}{ Community Periodontal Index $\ddagger$} \\
\hline Code 0,1 , or $2, \%$ & 39.5 & 25.7 & 23.2 & 29.1 & & $<0.001$ & $<0.001$ \\
\hline Code $3, \%$ & 40.3 & 48.8 & 43.6 & 37.3 & & & 0.657 \\
\hline Code $4, \%$ & 20.2 & 25.5 & 33.1 & 33.5 & & & $<0.001$ \\
\hline Age, years & $69.0(5.0)$ & $71.0(6.0)$ & $71.0(7.0)$ & $73.0(7.0)$ & $74.0(8.0)$ & $<0.001$ & $<0.001$ \\
\hline Female, $\%$ & 45.1 & 51.8 & 50.6 & 50.0 & 47.5 & 0.093 & 0.207 \\
\hline MMSE-total & $29.0(3.0)$ & $28.0(4.0)$ & $28.0(4.0)$ & $28.0(3.0)$ & $28.0(3.0)$ & 0.001 & $<0.001$ \\
\hline Recall score $2, \%$ & 21.3 & 24.8 & 24.8 & 27.9 & 32.2 & 0.016 & $<0.001$ \\
\hline GDS 6 or more, $\%$ & 9.9 & 11.3 & 15.3 & 13.1 & 15.3 & 0.049 & 0.007 \\
\hline Length of education, less than 12 years, $\%$ & 16.8 & 24.3 & 24.2 & 26.2 & 27.1 & $<0.001$ & $<0.001$ \\
\hline Alcohol intake, at least 1 day a week, $\%$ & 45.8 & 38.5 & 39.5 & 39.3 & 40.1 & 0.032 & 0.035 \\
\hline Current smoker, $\%$ & 6.7 & 7.4 & 12.7 & 12.7 & 10.7 & 0.001 & $<0.001$ \\
\hline Cancer, \% & 8.6 & 8.3 & 9.2 & 10.7 & 9.6 & 0.839 & 0.361 \\
\hline Myocardial infarction, $\%$ & 1.9 & 1.7 & 2.5 & 1.6 & 4.0 & 0.365 & 0.204 \\
\hline Cerebrovascular disease, $\%$ & 4.7 & 6.6 & 5.7 & 6.1 & 4.0 & 0.419 & 0.846 \\
\hline Diabetes mellitus, $\%$ & 11.5 & 15.1 & 17.2 & 13.1 & 16.9 & 0.052 & 0.039 \\
\hline Hypertension, \% & 66.7 & 69.4 & 73.6 & 72.1 & 70.1 & 0.171 & 0.054 \\
\hline Dyslipidemia, \% & 61.4 & 61.5 & 58.0 & 56.6 & 58.2 & 0.504 & 0.123 \\
\hline \multicolumn{8}{|l|}{ Follow-up characteristics } \\
\hline Systolic blood pressure, $\mathrm{mmHg}$ & $135.0(27.0)$ & $135.0(27.0)$ & $136.0(24.5)$ & $136.0(25.0)$ & $137.0(29.0)$ & 0.425 & 0.095 \\
\hline Diastolic blood pressure, $\mathrm{mmHg}$ & $72.0(15.0)$ & $73.0(16.0)$ & $71.0(16.0)$ & $71.0(15.0)$ & $71.0(16.0)$ & 0.033 & 0.002 \\
\hline $\begin{array}{l}\text { Cerebrovascular disease, } \% \\
\text { (Treatment started during the follow-up period) }\end{array}$ & 2.2 & 2.3 & 2.2 & 1.2 & 4.0 & 0.484 & 0.658 \\
\hline
\end{tabular}

Data are given as \% or median (interquartile range). ${ }^{*}$ Differences between the 5 groups were analyzed using the chi-square or Kruskal-Wallis test. ${ }^{\dagger}$ A trend test was performed using the Mantel-extension method or Jonckheere test. ${ }^{\ddagger}$ As the 177 edentulous subjects and 86 subjects who had 1-8 teeth had lost the teeth that were the targets of the examination, they were excluded from the analysis.

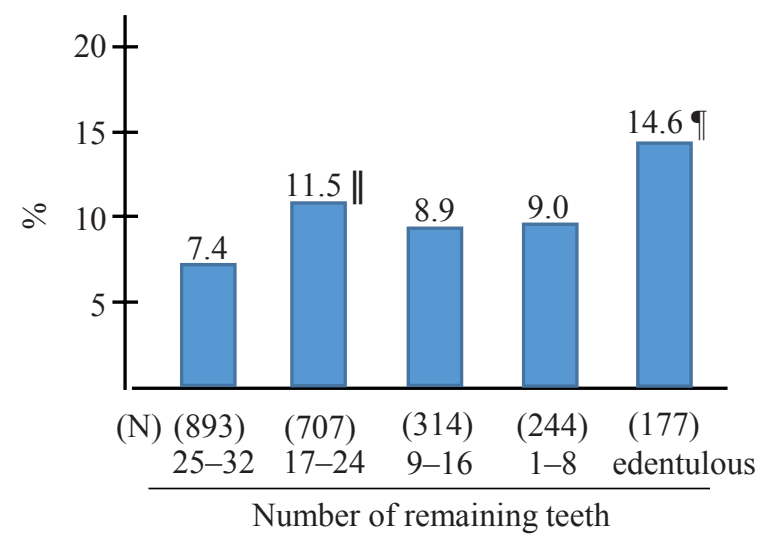

Fig. 2. Age- and gender-adjusted 5-year cumulative incidence of MMI according to the number of remaining teeth at baseline. Adjustments were made for age (65-69 years, 70-74 years, 75-79 years, 80-84 years, and 85 years or more) and gender by the direct method, and the data were tested for statistically significant differences by logistic regression analysis. ${ }^{\|} p<0.01$ and $\uparrow p<0.001$ versus $25-32$ remaining teeth.

condition), Recall score of 2 points, GDS score of 6 or more, education length less than 12 years, current smoker, and a positive history of diabetes mellitus.
Among the 2,335 individuals, 241 developed MMI. Fig. 2 shows the age- and gender-adjusted cumulative incidence of MMI according to the number of remaining teeth at baseline. The cumulative incidence was $11.5 \%$ in subjects with 17-24 remaining teeth and $14.6 \%$ in subjects without any teeth, showing significant differences compared to the $7.4 \%$ cumulative incidence of MMI in subjects with 25-32 remaining teeth.

Table 2 shows the multivariate-adjusted ORs for the development of MMI. After adjustment for the confounding factors in Model 2, the OR of per 1 tooth loss at baseline was 1.02 (95\% CI, 1.00-1.03). The ORs for the development of MMI in subjects with 17-24, $9-16,1-8$, and zero remaining teeth were $1.58(95 \%$ CI, 1.12-2.25), 1.17 (0.73-1.88), 1.08 (0.64-1.80), and 2.39 (1.48-3.86), respectively, compared to subjects with $25-32$ remaining teeth ( $p$ for trend $=0.020$ ). There were significant associations between having 17-24 remaining teeth and MMI, and total tooth loss and MMI. The management of hypertension and cerebrovascular disease during follow-up did not largely affect the association of tooth loss and MMI, 
Table 2

Multivariate-adjusted ORs for the development of MMI in 2,335 subjects during the 5-year follow-up peroid

\begin{tabular}{|c|c|c|c|c|}
\hline Dental variables & Model 1 & $P$ value & Model 2 & $P$ value \\
\hline Per 1 tooth loss at baseline & $1.01(1.00-1.03)$ & 0.051 & $1.02(1.00-1.03)$ & 0.039 \\
\hline \multicolumn{5}{|l|}{ Tooth category at baseline } \\
\hline $25-32$ & 1 & & 1 & \\
\hline $17-24$ & $1.57(1.11-2.23)$ & 0.011 & $1.58(1.12-2.25)$ & 0.010 \\
\hline $9-16$ & $1.15(0.72-1.84)$ & 0.550 & $1.17(0.73-1.88)$ & 0.527 \\
\hline $1-8$ & $1.06(0.63-1.76)$ & 0.834 & $1.08(0.64-1.80)$ & 0.781 \\
\hline Edentulous & $2.32(1.44-3.74)$ & 0.001 & $2.39(1.48-3.86)$ & $<0.001$ \\
\hline \multicolumn{5}{|c|}{ Community Periodontal Index at baseline* } \\
\hline Code $0-3$ & 1 & & 1 & \\
\hline Code 4 & $1.03(0.73-1.45)$ & 0.871 & $1.04(0.74-1.47)$ & 0.828 \\
\hline
\end{tabular}

Abbreviations: OR, odds ratio; CI, confidence interval. Values are expressed as OR (95\% CI). Dental variables were added separately to each model. Model 1: Adjusted for age (continuous variable), gender, MMSE-total, Recall, GDS, and education length. Model 2: Adjusted for the confounding factors used in Model 1, alcohol intake, smoking habits, history of cancer, myocardial infarction, cerebrovascular disease, diabetes mellitus, hypertension, and dyslipidemia, and follow-up characteristics (systolic and diastolic blood pressure and cerebrovascular disease). *As the 177 edentulous subjects and 86 subjects who had 1-8 teeth had lost the teeth that were the targets of the examination, they were excluded from the analysis.

Table 3

Association between the development of MMI and the decrease in the number of remaining teeth

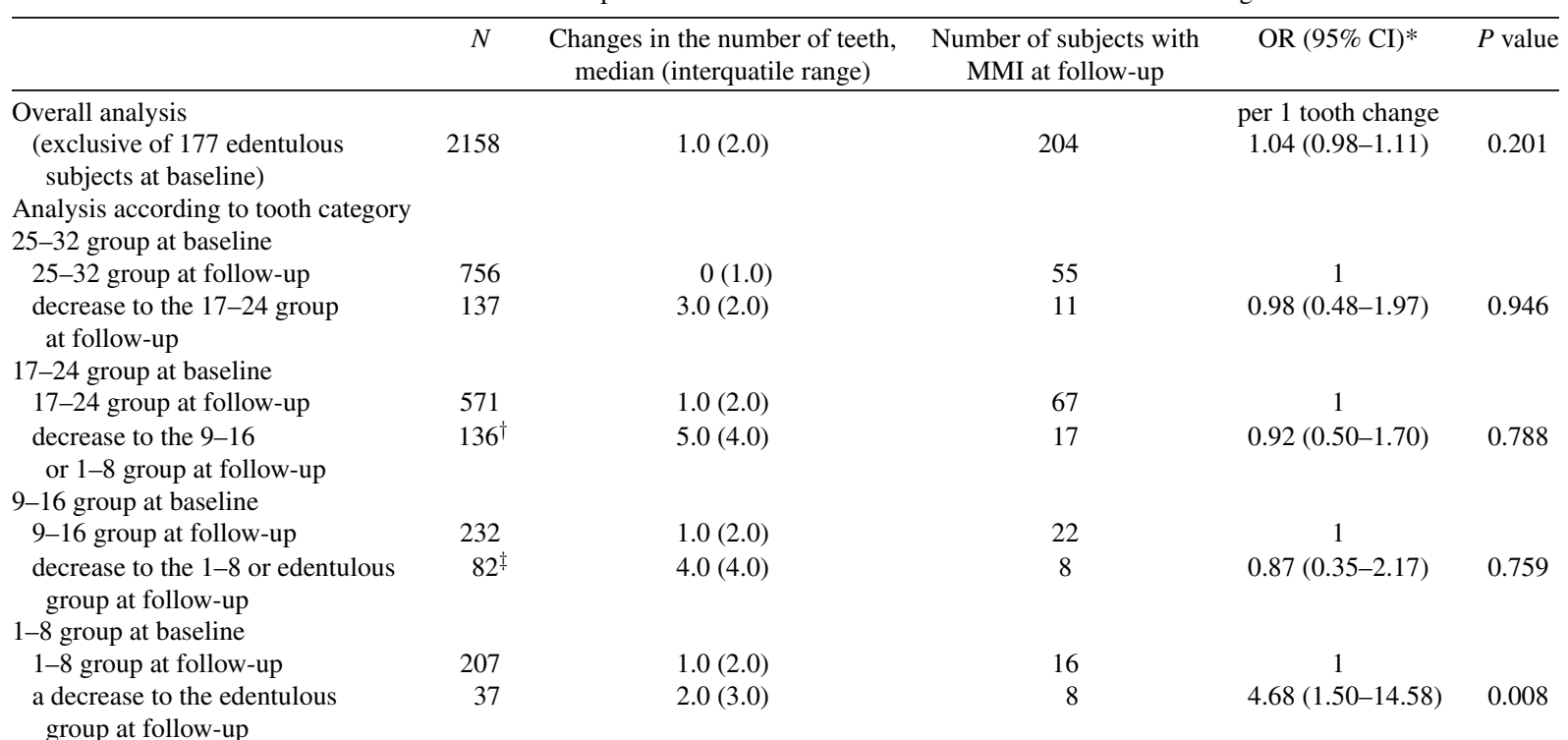

Changes in the number of teeth were calculated by baseline number minus follow-up number. A decrease of a tooth category was defined as a 1 or more decrease in tooth category at follow-up. *Adjusted for age (continuous variable), gender, MMSE-total, Recall, GDS, education length, alcohol intake, smoking habits, history of cancer, myocardial infarction, cerebrovascular disease, diabetes mellitus, hypertension, and dyslipidemia, and follow-up characteristics (systolic and diastolic blood pressure and cerebrovascular disease). ${ }^{\dagger}$ In 129 of the 136 subjects, the tooth category decreased to $9-16$, and it decreased to $1-8$ in the other 7 subjects. ${ }^{\ddagger}$ In 79 of the 82 subjects, the tooth category decreased to $1-8$, and the other 3 subjects had become edentulous.

considering the slight differences in the ORs between Models 1 and 2. The OR in subjects with CPI code 4 was $1.04(0.74-1.47)$ in Model 2 compared to those with code $0-3$. A significant association was not found between the CPI code and MMI.

Table 3 shows the effect of the decrease in the number of remaining teeth on the development of MMI. In the overall analysis, the OR of per 1 tooth change over 5 years was 1.04 (0.98-1.11), showing no significant association. No significant associations were found between the decrease of tooth category and MMI in the 25-32, 17-24, and 9-16 groups at baseline. The OR of becoming edentulous in the group with 1-8 teeth at baseline was 4.68 (1.50-14.58). A significant association was found between progressing to edentulism and the development of MMI. 


\section{DISCUSSION}

We observed a significant association between per 1 tooth loss at baseline, total tooth loss, progression to edentulism in the group with 1-8 remaining teeth, and the development of MMI, even after adjustment for confounding factors. A higher rate of tooth loss after baseline reportedly increased the risk of impaired cognitive performance in a follow-up study [14]. An approximately 5.5 year earlier decline to $50 \%$ of initial word recall status was observed for individuals with fewer teeth (less than 10) compared to individuals with 10 or more teeth, irrespective of apolipoprotein $\mathrm{E}$ (APOE) $\varepsilon 4$ status [15]. The results of the present study corroborate the results of these previous studies showing a significant association between tooth loss and cognitive dysfunction.

Several strengths of our study should be noted. First, we examined a causal relationship in a cohort study. Second, our use of MMI as an outcome variable is another strength. We speculate that we reduced the effect of the onset of cognitive impairment on the onset of tooth loss during this cohort study by excluding individuals with cognitive impairment at the time of the follow-up assessment from the analyses. We also counted subjects with cognitive impairment who were excluded from the analysis as part of the cohort, as shown in Supplementary Table 1. After adjustment for the confounding factors in Model 2, per 1 tooth loss at baseline showed a marginally significant relationship ( $p=0.063$ ). The ORs of $17-24$ and zero remaining teeth at baseline were 1.54 (95\% CI, 1.14-2.08) and $1.90(1.24-2.91)$ ( $\mathrm{p}$ for trend $=0.033)$. The differences in the observed results between Table 2 and Supplementary Table 1 were slight. Third, we examined whether changes in the number of teeth during the duration of the study increased the risk of MMI (Table 3). Within a limited duration of 5 years, we did not see an increase of risk of MMI in individuals with 9 or more teeth at baseline. However, we observed an increased risk of conversion from "cognitively intact" to MMI when individuals in the 1-8 group proceeded to edentulism due to additional tooth loss during the study.

The subjects that developed MMI in the current study maintained the basic and instrumental ADL at baseline. Therefore, there may be other biological bases different from a causal relationship in which a memory disorder induces poor oral conditions. Plausible biological explanations for the relationship between tooth loss and memory disorder include: 1) the inflammatory burden of periodontal disease; 2) genetic risk factors related to both periodontal disease and MMI; 3) a reduction of afferent input from sensory receptors; and 4) further confounding factors related to both tooth loss and MMI.

First, periodontal disease, which is a chronic infection of tissue surrounding the teeth by Gram-negative anaerobic bacteria, is the cause of a large number of missing teeth. Periodontal disease is related to systemic disease, such as carotid artery plaque [28], heart disease [29], and increased blood pressure [30]. Significant associations between the loss of periodontal attachment and worse cognitive function [31], and significant associations between the presence of antibodies against periodontal bacteria and poor delayed verbal recall [32] and the risk of $\mathrm{AD}[33,34]$ have also been reported in cross-sectional studies and a nested case-control study. Even if one loses teeth affected by periodontal disease, systemic damage may persist somewhat [28]. Inflammation has been implicated in the pathophysiology of $\mathrm{AD}[35,36]$; it has been hypothesized that inflammatory cytokines induced by periodontal infection and bacterial endotoxins can enter the brain and exacerbate a preexisting AD inflammatory pathology $[37,38]$.

Second, polymorphic cytokine genotypes may be related to periodontal disease and the risk of MMI. For example, interleukin (IL)-1A (-889) and IL-1B $(+3953)$ gene polymorphisms are reportedly associated with the severity of periodontal disease $[39,40]$ and the risk of $\mathrm{AD}[41,42]$.

Third, tooth loss or the reduction of mastication may affect the central nervous system. A study revealed a decrease in the number of choline acetyltransferasepositive neurons present in the nucleus of the diagonal band/medical septal nucleus in molar crown-cut off rats [43]. A decrease in the number of pyramidal cells was observed in the hippocampal CA1 and CA3 areas of molar-loss mice [44]. The number of high-affinity tyrosine kinase B mRNA-positive cells in the hippocampal CA3 area was negatively affected by the duration of tooth loss and the number of teeth extracted [45]. These findings are based on animal models, and further investigations in humans will be needed.

Fourth, tooth loss may induce nutritional deficits and, as a consequence, tooth loss may be associated with dementia [46]. Maintaining healthy vitamin C levels can have a protective function against age-related cognitive decline and AD [47]. Total tooth loss was associated with low citrus fruit consumption and low plasma vitamin C levels [48]. Low socio-economic status, negative events earlier in life, and head trauma with maxillofacial injuries may also be confounding factors related to tooth loss and memory disorders. 
A significant association was not seen between the CPI code and MMI in this study (Table 2). The CPI is suitable for the evaluation of current inflammatory swelling of the gingivae. Thus, the association between MMI status and the burden of past periodontal disease may be underestimated by this approach.

In Table 2, the ORs of 9-16 and 1-8 teeth for the development of MMI were not significant, but the OR of 17-24 teeth was significant. The proportion of subjects who had CPI code 3 or 4 (worse condition) was significantly higher in the 17-24 teeth group than in the 25-32 teeth group (74.3\% versus $60.5 \%$, respectively, $p<0.001$ ) (Table 1). The subjects in the 17-24 teeth group can be expected to have had larger numbers of teeth affected by periodontal disease than those in the 25-32 teeth group. Thus, the finding that the OR of the 17-24 teeth group was significant also supports the hypothesis that periodontal disease increases the risk of MMI.

Six limitations of the present study merit consideration. The primary limitation is that our results are biased by the exclusion of subjects who did not return for the follow-up examination. The proportions of edentulous individuals and individuals with baseline Recall scores of 2 points were greater in the excluded subjects. We speculate that a high-risk population for the development of MMI was excluded in this study. It is unclear whether the association between tooth loss and MMI was overestimated or underestimated. Second, the MMSE score depends on age and education. We might have defined some participants as cognitively intact, MMI, or cognitively impaired by mistake. Third, the CPI was selected as the index for evaluating periodontal disease. The CPI is sometimes used in epidemiologic surveys; however, it is difficult to evaluate cumulative inflammatory burden using the CPI. If cost and time limitations had permitted, radiographic evaluation of alveolar bone resorption of all teeth would have been more suitable. Fourth, we did not assess the extent to which dental caries or trauma accounted for tooth loss. Thus, we might have overestimated the relationship between periodontal disease and MMI. It would be practically difficult to conduct a dental chart review to verify the reasons for the loss of every tooth in each of the participants in their respective dental clinics. Fifth, we did not assess when the subjects lost their teeth. It is unclear how long it takes for MMI to develop after a decrease in the remaining number of teeth. Finally, APOE genotyping was not performed. APOE $\varepsilon 4$ carrier status was reportedly associated with lower scores on the delayed recall examination [49] and APOE $\varepsilon 4$ allele frequency was significantly higher in patients with early stage cognitive impairment versus healthy controls [50]. Considering the presence of APOE $\varepsilon 4$ carriers in the MMI group, our findings might have overestimated the relationship between tooth loss and MMI.

Within the limitations of the study design described above, the results of this study showed that tooth loss may be an independent risk factor for MMI. Preventive measures against tooth loss at a younger age may be effective at reducing the risk of memory disorders.

\section{ACKNOWLEDGMENTS}

The authors express their sincere gratitude to all participants for their cooperation in this study and the staff of the Fujiwara-kyo study.

This work was supported by a Grant-in-Aid for Young Scientists (No. 22790566:2010-2013) from the Japanese Ministry of Education, Culture, Sports, Science, and Technology, by a research grant J110400001 from Nara Medical University (2007-2012), and by a research grant from the Mitsui Sumitomo Insurance Welfare Foundation (2007).

Authors' disclosures available online (http://www.jalz.com/disclosures/view.php?id=2564 ).

\section{SUPPLEMENTARY MATERIAL}

The supplementary material is available in the electronic version of this article: http://dx.doi.org/ 10.3233/JAD-141665.

\section{REFERENCES}

[1] Gatz M, Mortimer JA, Fratiglioni L, Johansson B, Berg S, Reynolds CA, Pedersen NL (2006) Potentially modifiable risk factors for dementia in identical twins. Alzheimers Dement 2 , 110-117.

[2] Stein PS, Desrosiers M, Donegan SJ, Yepes JF, Kryscio RJ (2007) Tooth loss, dementia and neuropathology in the Nun Study. J Am Dent Assoc 138, 1314-1322.

[3] Batty GD, Li Q, Huxley R, Zoungas S, Taylor BA, Neal B, de Golan B, Woodward M, Harrap SB, Colagiuri S, Patal A, Chalmers J, On behalf of the ADVANCE Collaborative group (2013) Oral disease in relation to future risk of dementia and cognitive decline: Prospective cohort study based on the action in diabetes and vascular disease: Preterax and diamicron modified-release controlled evaluation (ADVANCE) trial. Eur Psychiatry 28, 49-52.

[4] Bergdahl M, Habib R, Bergdahl J, Nyberg L, Nilsson L-G (2007) Natural teeth and cognitive function in humans. Scand J Psychol 48, 557-565.

[5] Kamer AR, Morse DE, Holm-Pedersen P, Mortensen EL, Avlund K (2012) Periodontal inflammation in relation to cognitive function in an older adult Danish population. J Alzheimers Dis 28, 613-624. 
[6] Hansson P, Sunnegårdh-Grönberg K, Bergdahl J, Bergdahl M, Nyberg L, Nilsson LG (2013) Relationship between natural teeth and memory in a healthy elderly population. Eur J Oral Sci 121, 333-340.

[7] Humphrey LL, Fu R, Buckley DI, Freeman M, Helfand M (2008) Periodontal disease and coronary heart disease incidence: A systematic review and meta-analysis. J Gen Intern Med 23, 2079-2086.

[8] Kamer AR, Craig RG, Dasanayake AP, Brys M, GlodzikSobanska L, de Leon MJ (2008) Inflammation and Alzheimer's disease: Possible role of periodontal diseases. Alzheimers Dement 4, 242-250.

[9] Ishikawa T, Ikeda M, Matsumoto N, Shigenobu K, Brayne C, Tanabe H (2006) A longitudinal study regarding conversion from mild memory impairment to dementia in a Japanese community. Int J Geriatr Psychiatry 21, 134-139.

[10] Sonobe N, Hata R, Ishikawa T, Sonobe K, Matsumoto T, Toyota Y, Mori T, Fukuhara R, Komori K, Ueno S, Tanimukai S, Ikeda M (2011) Risk of progression from mild memory impairment to clinically diagnosable Alzheimer's disease in a Japanese community (from the Nakayama Study). Int Psychogeriatr 23, 772-779.

[11] Petersen RC, Smith GE, Waring SC, Ivnik RJ, Tangalos EG, Kokmen E (1999) Mild congnitive impairment. Arch Neurol 56, 303-308.

[12] Okamoto N, Morikawa M, Okamoto K, Habu N, Hazaki K, Harano A, Iwamoto J, Tomioka K, Saeki K, Kurumatani N (2010) Tooth loss is associated with mild memory impairment in the elderly: The Fujiwara-kyo study. Brain Res 1349 68-75.

[13] Okamoto N, Morikawa M, Okamoto K, Habu N, Iwamoto J, Tomioka K, Saeki K, Yanagi M, Amano N, Kurumatani N (2010) Relationship of tooth loss to mild memory impairment and cognitive impairment: Findings from the fujiwara-kyo study. Behav Brain Funct 6,77.

[14] Kaye EK, Valencia A, Baba N, Spiro A, Dietrich T, Garcia RI (2010) Tooth loss and periodontal disease predict poor cognitive function in older men. J Am Geriatr Soc 58, 713718.

[15] Stein PS, Kryscio RJ, Desrosiers M, Donegan SJ, Gibbs MB (2010) Tooth loss, apolipoprotein E, and decline in Delayed Word Recall. J Dent Res 89, 473-477.

[16] Okamoto N, Tomioka K, Saeki K, Iwamoto J, Morikawa M, Harano A, Kurumatani N (2012) Relationship between swallowing problems and tooth loss in community-dwelling independent elderly adults: The Fujiwara-kyo Study. J Am Geriatr Soc 60, 849-853.

[17] Morikawa M, Okamoto N, Kiuchi K, Tomioka K, Iwamoto J, Harano A, Saeki K, Fukusumi M, Hashimoto K, Amano N, Hazaki K, Yanagi M, Iki M, Yamada F, Kishimoto T, Kurumatani N (2013) Association between depressive symptoms and metabolic syndrome in Japanese community-dwelling older people: A cross-sectional analysis from the baseline results of the Fujiwara-kyo prospective cohort study. Int J Geriatr Psychiatry 28, 1251-1259.

[18] Ishizaki J, Meguro K, Ambo H, Shimada M, Yamaguchi S, Hayasaka C, Komatsu H, Sekita Y, Yamadori A (1998) A normative, community-based study of Mini-Mental State in elderly adults: The effect of age and educational level. J Gerontol B Psychol Sci Soc Sci 53, P359-363.

[19] American Psychiatric Association (1987) Diagnostic and Statistical Manual of Mental Disorders. 3rd ed rev. American Psychiatric Association, Washington, DC.

[20] Ishizaki T, Watanabe S, Suzuki T, Shibata H, Haga H (2000) Predictors for functional decline among nondisabled older
Japanese living in a community during a 3-year follow-up. J Am Geriatr Soc 48, 1424-1429.

[21] Schreiner AS, Hayakawa H, Morimoto T, Kakuma T (2003) Screening for late life depression: Cut-off scores for the Geriatric Depression Scale and the Cornell Scale for depression in dementia among Japanese subjects. Int J Geriatr Psychiatry 18, 498-505.

[22] Meguro K, Ishii H, Yamaguchi S, Ishizaki J, Sato M, Hashimoto R, Meguro M, Lee E, Tanaka Y, Kasuya M, Sekita Y (2004) Prevalence and cognitive performances of clinical dementia rating 0.5 and mild cognitive impairment in Japan: The Tajiri project. Alzheimer Dis Assoc Disord 18, 3-10.

[23] Sasaki M, Kodama C, Hidaka S, Yamashita F, Kinoshita T, Nemoto K, Ikejima C, Asada T (2009) Prevalence of four subtypes of mild cognitive impairment and APOE in a Japanese community. Int J Geriatr Psychiatry 24, 1119-1126.

[24] World Health Organization (1997) Oral health surveys-Basic Methods, 4th ed. Geneva, pp. 36-38.

[25] Seino Y, Nanjo K, Tajima N, Kadowaki T, Kashiwagi A, Araki E, Ito C, Inagaki N, Iwamoto Y, Kasuga M, Hanafusa T, Haneda M, Ueki K (2012) Report of the committee on the classification and diagnostic criteria of diabetes mellitus (revision for international harmonization of HbA1c in Japan). J Jpn Diabetes Soc 55, 485-504.

[26] Ogihara T, Kikuchi K, Matsuoka H, Fujita T, Higaki J, Horiuchi M, on behalf of the Japanese Society of Hypertension Committee (2009) The Japanese Society of Hypertension Guidelines for the Management of Hypertension (JSH2009). Hypertens Res 32, 3-107.

[27] Teramoto T, Sasaki J, Ueshima H, Egusa G, Kinoshita M, Shimamoto K, Daida H, Biro S, Hirobe K, Funahashi T, Yokote K, Yokote M (2007) Diagnostic criteria for dyslipidemia: Executive summary of Japan Atherosclerosis Society (JAS) guideline for diagnosis and prevention of atherosclerotic cardiovascular diseases for Japanese. J Atheroscler Thromb 14, 155-158.

[28] Desvarieux M, Demmer RT, Rundek T, Boden-Albala B, Jacobs DR, Papapanou PN, Sacco RL (2003) Relationship between periodontal disease, tooth loss, and carotid artery plaque: The Oral Infections and Vascular Disease Epidemiology Study (INVEST). Stroke 34, 2120-2125.

[29] Okoro CA, Balluz LS, Eke PI, Ajani UA, Strine TW, Town M, Mensah GA, Mokdad AH (2005) Tooth loss and heart disease: Findings from the behavioral risk factor surveillance system. Am J Prev Med 29, 50-56.

[30] Peres MA, Tsakos G, Barbato PR, Silva DAS, Peres KG (2012) Tooth loss is associated with increased blood pressure in adults: A multidisciplinary population-based study. $J$ Clin Periodontol 39, 824-833.

[31] Stewart R, Sabbah W, Tsakos G, D'Aiuto F, Watt RG (2008) Oral health and cognitive function in the Third National Health and Nutrition Examination Survey (NHANES III). Psychosom Med 70, 936-941.

[32] Noble JM, Borrell LN, Papapanou PN, Elkind MSV, Scarmeas N, Wright CB (2009) Periodontitis is associated with cognitive impairment among older adults: Analysis of NHANES-III. J Neurol Neurosurg Psychiatry 80, 1206-1211.

[33] Kamer AR, Craig RG, Pirraglia E, Dasanayake AP, Norman RG, Boylan RJ, Nehorayoff A, Glodzik L, Brys M, de Leon MJ (2009) TNF- $\alpha$ and antibodies to periodontal bacteria discriminate between Alzheimer's disease patients and normal subjects. J Neuroimmunol 216, 92-97.

[34] Stein PS, Steffen MJ, Smith C, Jicha G, Ebersole JL, Abner E, Dawson III D (2012) Serum antibodies to periodontal 
pathogens are a risk factor for Alzheimer's disease. Alzheimers Dement 8, 196-203.

[35] Blasko I, Grubeck-Loebenstein B (2003) Role of the immune system in the pathogenesis, prevention and treatment of Alzheimer's disease. Drugs Aging 20, 101-113.

[36] Sastre M, Walter J, Gentleman SM (2008) Interactions between APP secretases and inflammatory mediators. $\mathrm{J} \mathrm{Neu}$ roinflammation $\mathbf{5}, 25$.

[37] Watts A, Crimmins EM, Gatz M (2008) Inflammation as a potential mediator for the association between periodontal disease and Alzheimer's disease. Neuropsychiatr Dis Treat 4, 865-876.

[38] Poole S, Shinghrao SK, Kesavalu L, Curtis MA, Crean S (2013) Determining the presence of periodontopathic virulence factors in short-term postmortem Alzheimer's disease brain tissue. J Alzheimers Dis 36, 665-677.

[39] Kornman KS, Crane A, Wang H-Y, di Giovine FS, Newman MG, Pirk FW, Wilson Jr TG, Higginbottom FL, Duff GW (1997) The interleukin-1 genotype as a severity factor in adult periodontal disease. J Clin Periodontol 24, 72-77.

[40] Galbraith GMP, Hendley TM, Sanders JJ, Palesch Y, Pandey JP (1999) Polymorphic cytokine genotypes as markers of disease severity in adult periodontitis. J Clin Periodontol 26, 705-709.

[41] Nicoll JAR, Mrak RE, Graham DI, Stewart J, Wilcock G, MacGowan S, Esiri MM, Murray LS, Dewar D, Love S, Moss T, Griffin WST (2000) Association of interleukin-1 gene polymorphisms with Alzheimer's disease. Ann Neurol 47, 365-368.

[42] McGeer PL, McGeer EG (2001) Polymorphisms in inflammatory genes and the risk of Alzheimer disease. Arch Neurol 58, 1790-1792.
[43] Terasawa H, Hirai T, Ninomiya T, Ikeda Y, Ishijima T, Yajima T, Hamaue N, Nagase Y, Kang Y, Minami M (2002) Influence of tooth-loss and concomitant masticatory alterations on cholinergic neurons in rats: Immunohistochemical and biochemical studies. Neurosci Res 43, 373-379.

[44] Oue H, Miyamoto Y, Okada S, Koretake K, Jung C-G, Michikawa M, Akagawa Y (2013) Tooth loss induces memory impairment and neuronal cell loss in APP transgenic mice. Behav Brain Res 252, 318-325.

[45] Yamazaki K, Wakabayashi N, Kobayashi T, Suzuki T (2008) Effect of tooth loss on spatial memory and TrkB-mRNA levels in rats. Hippocampus $\mathbf{1 8}, 542-547$.

[46] Kim J-M, Stewart R, Prince M, Kim S-W, Yang S-J, Shin II-S, Yoon JS (2007) Dental health, nutritional status and recent-onset dementia in a Korean community population. Int $J$ Geriatr Psychiatry 22, 850-855.

[47] Harrison FE (2012) A critical review of vitamin C for the prevention of age-related cognitive decline and Alzheimer's disease. J Alzheimers Dis 29, 711-726.

[48] Lowe G, Woodward M, Rumley A, Morrison C, TunstallPedoe H, Stephen K (2003) Total tooth loss and prevalent cardiovascular disease in men and women: Possible roles of citrus fruit consumption, vitamin $\mathrm{C}$, and inflammatory and thrombotic variables. J Clin Epidemiol 56, 694-700.

[49] O'Hara R, Yesavage JA, Kraemer HC, Mauricio M, Friedman LF, Murphy Jr GM (1998) The APOE $\varepsilon 4$ allele is associated with decline on delayed recall performance in communitydwelling older adults. J Am Geriatr Soc 46, 1493-1498.

[50] Dubé JB, Johansen CT, Robinson JF, Lindsay J, Hachinski V, Hegele RA (2013) Genetic Determinants of "Cognitive impairment, no dementia”. J Alzheimers Dis 33, 831-840. 